\title{
Energy prices in the presence of plant indivisibilities
}

\author{
Ronald Fischer \\ Pablo Serra * This research was supported by FONDECYT project \#1010520.
}

October 3, 2001

\begin{abstract}
In several countries (Chile, Bolivia, Argentina and Peru, among others), power plants are dispatched according to merit order, i.e., based on the marginal operating costs of the plants. In this scheme, the plant with the highest marginal cost sets the spot price at which firms trade the energy requires to fulfill their contracts. The model assumes that plants can operate at any level up to capacity, whereas real power plants have minimum operating levels. This implies that a low cost plant might have to reduce its supply in order to accommodate the minimum operating level of a more expensive power plant. This paper derives the welfare maximizing price rules in this case and shows that the standard peak load pricing rules no longer apply.
\end{abstract}

JEL Classification: L51, L94.

KEYwords: Peak load pricing, indivisibility, electricity.

*The authors are at the University of Chile. Address: Center for Applied Economics (CEA), Department of Industrial Engineering, University of Chile, Av. República 701, Santiago, Chile. E-mail:rfischer@dii.uchile.cl,pserra@ dii.uchile.cl 


\section{Introduction}

Earlier deregulators of electric industries such as Chile, Bolivia and Peru adopted the dispatch and pricing system developed by Electricite de France (EDF). ${ }^{1}$ In this approach demand is assumed to be unresponsive to price, hence the role of the systems operator is to accommodate power supply to the fixed demand. Plants are dispatched according to the merit order, i.e., they are ranked according to their marginal operating costs and dispatched in ascending order until demand is satisfied at the given price. The pricing system has two components. First, plants are paid the price of energy for their production, which is given by the marginal cost of the most expensive plant in operation. ${ }^{2}$ In addition, each plant receives a capacity payment equal to the energy it delivers at peak demand times a capacity charge which equals the cost of the least expensive means of expanding capacity. Assuming that demand is inelastic, plants are divisible and no uncertainty, it can be shown that this system of rewards known as peak-load pricing satisfies the following three desirable properties (see Turvey (1968)):

- Any plant that is dispatched by the system operator obeys willingly.

- Each plant pays for its operation and investment costs.

- The rules of dispatch minimize the long and short term cost of providing electricity.

These conditions imply that the system can be decentralized, i.e., given these rules of operation, the market will provide the optimal investment mix that replicates the planner's solution. There have been many extensions of peak-load pricing in order to adapt these results to the real world. Most researchers have explored the consequences of eliminating the assumption of no uncertainty. ${ }^{3}$ Less work has been done on the effects of eliminating the assumption of perfect divisibility of power plants.

In this paper we focus on the effects of minimum operational levels (MOLs) below which some plants cannot operate. This raises the possibility that within certain demand ranges it might be necessary to reduce the supply of a low operating cost plant, which is replaced by the output from a higher operating cost plant pinned at its MOL, in order to adjust supply to demand in real time. ${ }^{4}$ We analyze the properties of standard peak-load pricing in the presence of MOLs, as we are interested in finding the reward structure that will provide the welfare maximizing short and long term signals to generating firms. ${ }^{5}$

Even though system operators are used to dealing with these problems by using large linear programming models to find the dispatch schedule that achieves the minimum operational cost,

\footnotetext{
${ }^{1}$ See Joskow (1976) for a description of the EDF system.

${ }^{2}$ To be precise, the marginal cost of energy is normally used only for transactions between generating companies so that they can satisfy their energy contracts with clients.

${ }^{3}$ Under conditions of supply uncertainty, it is necessary to include an outage cost. For an extension with uncertainty in demand and supply, see Chao (1983).

${ }^{4}$ Other cases in which plants with higher operating costs displace plants with lower operating costs occur when the former have long ramp up periods so it is not economical to make them run only at peak time or when the former are forced to operate in order to maintain the integrity of the system.

${ }^{5}$ The lack of explicit rules leads to implicit contracts between participants. Unfortunately, the informal nature of these arrangements is a barrier to new entrants.
} 
there have been no theoretical analysis of the pricing system that provides the welfare maximizing short and long-run signals to generating companies. A possible explanation is that in state owned monopolies the assignment of revenues to specific plants is not an issue and because many countries that have deregulated (and privatized) their industry use bid markets, an environment in which the problems caused by indivisibilities are less relevant. ${ }^{6}$ Moreover, these problems are of less importance in large systems in which plants are small in relation to the size of the system.

We model a simple case with two types of plants, in order to highlight our results. The first type of plant has a high investment cost but a low operating cost as compared to the second type of plant. We examine the case in which a high operating cost plant operating at its MOL displaces part of the production of a low operating cost plant. Hence the marginal cost of the system corresponds to that of the displaced low operating cost plant, as this is the one that absorbs small demand fluctuations. The problem is that if we set price equal to this marginal cost, the high operating cost plant makes losses, violating the first optimal property of peak load pricing. We show that keeping marginal cost pricing requires capacity charges above the cost of expansion, because MOLs increase the total cost of the system.

Note that with MOLs the simplicity of the peak-loading pricing rule is lost. First, on occasion the high operating cost plant must be compelled to operate, violating the condition that any plant that is dispatched by the system operator obeys willingly. Second, when capacity charges exceed the cost of expansion, there are incentives for an entrant to supply all demand at peak load using a high operating cost plant, since it receives the cost of capacity plus the cost of power and an additional amount that allows it to earn rents. However, this means that there is no supply in low demand periods (or supply is provided only with high cost plants) since low operating cost plants would have losses. Hence, implementation of peak-load pricing in the presence of MOLs requires that the system operator pay capacity payments only to those plants that generate whenever it is requested. In addition, a mayor problem with MOLs lies in the complexity of the solution, since the system operator must perform the computations needed to derive the optimal rewards, which lacks the transparency of the rewards in the idealized peak load pricing model.

In the remainder of this paper we formalize these results in a simple model. In particular, we find the power payments that lead to the correct short and long term operation when there are indivisibilities. ${ }^{7}$

\section{The model}

In order to simplify the exposition we assume that there are two types of plants. Type 1 plants have lower operating cost but higher investments cost than type 2 plants. Hence, in general the former operate as base load plants while the second type operates at peak time. Operating costs

\footnotetext{
${ }^{6}$ In the bidding approach (developed first in the UK, and used in many European countries, Colombia, and some states in the US) generating firms make bids on the amounts and the prices of electricity they are willing to provide the system. The system operator uses these bids to construct an anergy supply function and sets the dispatch order. The importance of MOLs in market based systems should be smaller, since firm's bids should compensate for these problems.

${ }^{7}$ The indivisibilities discussed in this paper are different from those studied in the early paper by Williamson (1966). In that paper, indivisibility meant that plants had a fixed minimum size, but it could produce at any power level. In the type of indivisibility considered in this paper, the plant cannot operate below a certain power level.
} 


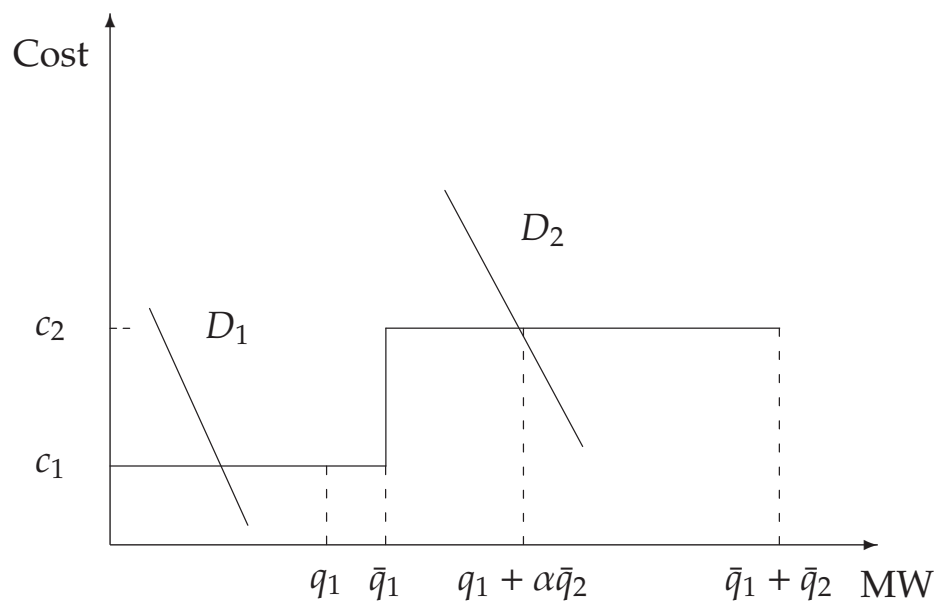

Figure 1: The problem of minimum operating levels

are $c_{1}<c_{2}$ and the unit costs of capacity are given by $f_{1}>f_{2}$. The generating capacities of the two types of plants are given by $\bar{q}_{1} \mathrm{y} \bar{q}_{2}$. We assume the existence of a minimum size for type 2 plants, and that demand justifies the installation of one plant. This plant has a minimum operating level of $\alpha \bar{q}_{2}$, with $0 \leq \alpha \leq 1$.

Consider the case in which the existence of the minimum operating level in plant type 2 alters the merit order of dispatch within some range of demand. For example, in order to satisfy an increase in demand, the system operator may be forced to dispatch the type 2 plant. Because it has a MOL, its entry displaces part of the capacity supplied by a lower cost type 1 plant. Figure 1 shows the change in the dispatch order when demand increases from $D_{1}$ to $D_{2}$. Suppose that at price $c_{2}$, demand is less than $\bar{q}_{1}+\alpha \bar{q}_{2}$, but higher than $\bar{q}_{1}$. When demand is given by curve $D_{2}$, the type 2 plant must enter in order to supply the market, but the MOL displaces some of the capacity of the type 1 plant, which produces at a level $q_{1}<\bar{q}_{1}$.

The load duration curve described in figure 2 orders the 8760 hours of the year according to the demand for energy, which is assumed inelastic. Let $D$ denote the maximum demand, $q(t)$ demand in the $t$-th hour with highest demand, and $t(q)$ its inverse. For simplicity we assume that function $t$ is differentiable, hence $t^{\prime}(q) \leq 0$. In what follows we use the following notation: $T=8760$, $\bar{q}_{0}=\bar{q}_{1}+\alpha \bar{q}_{2}, T_{0}=t\left(\bar{q}_{0}\right)$, and $T_{1}=t\left(\bar{q}_{1}\right)$. In the figure, during the $\left(T-T_{1}\right)$ hours of low demand only the type 1 plants operate and spot price equals $c_{1}$. At $T_{1}$, these plants are operating at full capacity and the type 2 plant must begin to provide energy. Given that the type 2 plant is pinned by its MOL, the type 1 plants must cut back their supply. This inversion of the normal merit order occurs in the range $\overline{T_{0} T_{1}}$. At $T_{0}$, demand is such that the type 1 plants are operating at full capacity and the type 2 plant needs to generate above its MOL, so the spot price is given by the cost of the type 2 plant.

Result 1 A MOL leads to an inversion of the merit order of dispatch. 


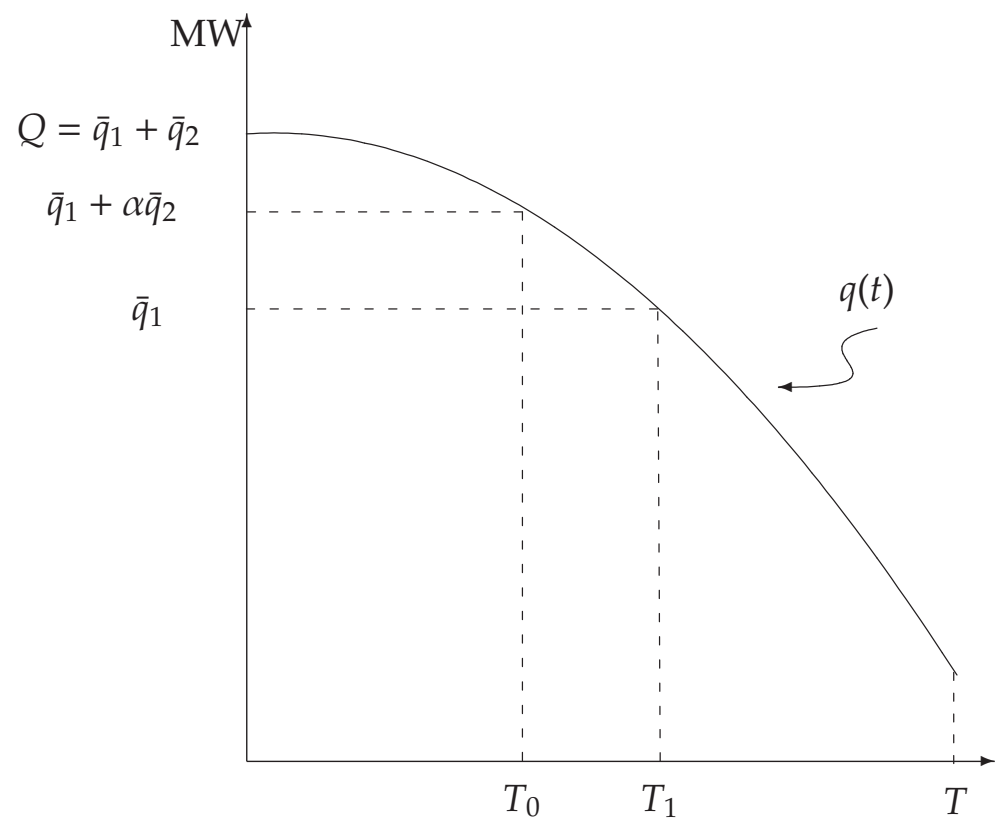

Figure 2: A Load duration curve with indivisibilities

\subsection{The case with no MOLs}

When there are no indivisibilities $\alpha=0$, i.e., $T_{0}=T_{1}$, then the total cost of plants (investment plus operational cost) are: ${ }^{8}$

$$
\begin{aligned}
& C_{1}=c_{1} \int_{T_{1}}^{T} q(t) d t+c_{1} \int_{0}^{T_{1}} \bar{q}_{1} d t+f_{1} \bar{q}_{1} \\
& c_{2}=c_{2} \int_{0}^{T_{1}}\left\{q(t)-\bar{q}_{1}\right\} d t+f_{2} \bar{q}_{2}
\end{aligned}
$$

We consider a classical peak-load pricing system.Hence plants receive a payment per unit of energy equal to the marginal cost of energy. They also receive a capacity payment equal to the marginal investment cost in type 2 plants $f_{2}$. The revenues accruing to each type (including the capacity charges) are:

$$
\begin{aligned}
& R_{1}=c_{1} \int_{T_{1}}^{T} q(t) d t+c_{1} \int_{0}^{T_{1}} \bar{q}_{1} d t+f_{2} \bar{q}_{1} \\
& R_{2}=c_{2} \int_{0}^{T_{1}}\left\{q(t)-\bar{q}_{1}\right\} d t+f_{2} \bar{q}_{2}
\end{aligned}
$$

\footnotetext{
${ }^{8}$ See Boiteux (1960) for the earliest analysis of the peak-load problem.
} 
Clearly, the type 2 plants are in a zero-profit equilibrium in all assignments. The type 1 plants are in equilibrium when $R_{1}=C_{1}$, which implies that

$$
T_{1}=\frac{f_{1}-f_{2}}{c_{2}-c_{1}}
$$

Then the assignment of capacity to type 1 plants is determined using the load duration curve. If $T_{1} \leq T$, then $\bar{q}_{1}=q\left(T_{1}\right)$, otherwise $\bar{q}_{1}=0$. Boiteux (1960) shows that this decentralized equilibrium is optimal as it minimizes the total cost of the system.

\subsection{The general case with MOLs}

When $\alpha \neq 0$, we have that $T_{0}<T_{1}$. In the interval $\overline{T_{0} T_{1}}$, type 1 plants do not operate at full capacity. Hence, assuming that $\bar{q}_{1} \geq \alpha \bar{q}_{2}$ the total cost of each plant is:

$$
\begin{aligned}
& C_{1}=c_{1} \int_{0}^{T_{0}} \bar{q}_{1} d t+c_{1} \int_{T_{0}}^{T_{1}}\left\{q(t)-\alpha \bar{q}_{2}\right\} d t+c_{1} \int_{T_{1}}^{T} q(t) d t+f_{1} \bar{q}_{1} \\
& C_{2}=c_{2} \int_{0}^{T_{0}}\left\{q(t)-\bar{q}_{1}\right\} d t+c_{2} \int_{T_{0}}^{T_{1}} \alpha \bar{q}_{2} d t+f_{2} \bar{q}_{2}
\end{aligned}
$$

Then the total cost of the system is:

$$
C=C_{1}+C_{2}=c_{1} \int_{0}^{T} q(t) d t+\left(c_{2}-c_{1}\right)\left[\int_{0}^{T_{0}}\left(q(t)-\bar{q}_{1}\right) d t+\int_{T_{0}}^{T_{1}} \alpha \bar{q}_{2} d t\right]+f_{1} \bar{q}_{1}+f_{2} \bar{q}_{2}
$$

Next we derive the capacities $\bar{q}_{1}$ and $\bar{q}_{2}$ that minimize the total cost of the system and satisfy demand. Hence we need to impose the restriction $\bar{q}_{1}+\bar{q}_{2} \geq D$, that is, total capacity must exceed peak demand $D$. In the previous analysis we implicitly imposed the condition that the planner does not operate plant 2 unless necessary, but this was not introduced as a constraint in the maximization problem. We generalize the presentation and assume that the central planner also decides the time $T_{1}$, at which it begins operating the type 2 plant. Thus we need to impose the constraint $q\left(T_{1}\right) \leq \bar{q}_{1}$, that is, at the time at which plant 1 has to reduce its load in order to accommodate the entry of plant 2 , demand must be lower or equal to the capacity of the type 1 plant. Letting $\lambda$ and $\mu$ be the Lagrange multipliers associated to the first and second restriction respectively, the Lagrange function equals:

$$
\mathcal{L}=c_{1} \int_{0}^{T} q(t) d t+\left(c_{2}-c_{1}\right)\left[\int_{0}^{T_{0}}\left(q(t)-\bar{q}_{1}\right) d t+\int_{T_{0}}^{T_{1}} \alpha \bar{q}_{2} d t\right]+f_{1} \bar{q}_{1}+f_{2} \bar{q}_{2}+\lambda\left(D-\bar{q}_{1}-\bar{q}_{2}\right)+\mu\left(q\left(T_{1}\right)-\bar{q}_{1}\right)
$$

The resulting Kuhn-Tucker conditions are:

$$
\frac{\partial \mathcal{L}}{\partial \bar{q}_{1}}=\left(c_{2}-c_{1}\right)\left[-T_{0}+\left(q\left(T_{0}\right)-\bar{q}_{1}-\alpha \bar{q}_{2}\right) \frac{d T_{0}}{d \bar{q}_{1}}\right]+f_{1}-\lambda-\mu \geq 0, \bar{q}_{1} \frac{\partial \mathcal{L}}{\partial \bar{q}_{1}}=0
$$




$$
\begin{aligned}
& \frac{\partial \mathcal{L}}{\partial \bar{q}_{2}}=\left(c_{2}-c_{1}\right)\left[\left(q\left(T_{0}\right)-\bar{q}_{1}-\alpha \bar{q}_{2}\right) \frac{d T_{0}}{d \bar{q}_{1}}+\alpha\left(T_{1}-T_{0}\right)\right]+f_{2}-\lambda \geq 0, \bar{q}_{2} \frac{\partial \mathcal{L}}{\partial \bar{q}_{2}}=0 \\
& \frac{\partial \mathcal{L}}{\partial T_{1}}=\left(c_{2}-c_{1}\right)\left[\alpha \bar{q}_{2}+\left(q\left(T_{0}\right)-\bar{q}_{1}-\alpha \bar{q}_{2}\right) \frac{d T_{0}}{d T_{1}}\right]+\mu q^{\prime}\left(T_{1}\right) \geq 0, T_{1} \frac{\partial \mathcal{L}}{\partial T_{1}}=0 \\
& \frac{\partial \mathcal{L}}{\partial \lambda}=D-\bar{q}_{1}-\bar{q}_{2} \leq 0, \lambda \frac{\partial \mathcal{L}}{\partial \lambda}=0 \\
& \frac{\partial \mathcal{L}}{\partial \mu}=q\left(T_{1}\right)-\bar{q}_{1} \leq 0, \mu \frac{\partial \mathcal{L}}{\partial \mu}=0
\end{aligned}
$$

The first three inequalities can be written as:

$$
\begin{aligned}
& \frac{\partial \mathcal{L}}{\partial \bar{q}_{1}}=\left(c_{2}-c_{1}\right) T_{0}+f_{1}-\lambda-\mu \geq 0, q_{1} \frac{\partial \mathcal{L}}{\partial \bar{q}_{1}}=0 \\
& \frac{\partial \mathcal{L}}{\partial \bar{q}_{2}}=\left(c_{2}-c_{1}\right) \alpha\left(T_{1}-T_{0}\right)+f_{2}-\lambda \geq 0, q_{2} \frac{\partial \mathcal{L}}{\partial \bar{q}_{2}}=0 \\
& \frac{\partial \mathcal{L}}{\partial T_{1}}=\left(c_{2}-c_{1}\right) \alpha \bar{q}_{2}+\mu q^{\prime}\left(T_{1}\right) \geq 0, T_{1} \frac{\partial \mathcal{L}}{\partial T_{1}}=0
\end{aligned}
$$

Assuming an interior solution $\bar{q}_{2}, \bar{q}_{1}>0$, the first two inequalities become equalities. From equation (9) follows that $\lambda>0$ and from equation (6) we have that $D=\bar{q}_{1}+\bar{q}_{2}$ and therefore $D=q(0)>\bar{q}_{1}$. Therefore, from equation (7) we have $T_{1}>0$. Thus equation (10) implies $\mu>0$, from which equation (7) is an equality and $T_{1}=t\left(\bar{q}_{1}\right)$. Finally, equation (10) can be rewritten: ${ }^{9}$

$$
t^{\prime}\left(\bar{q}_{1}\right) \frac{\partial \mathcal{L}}{\partial T_{1}}=\left(c_{2}-c_{1}\right) \alpha \bar{q}_{2} t^{\prime}\left(\bar{q}_{1}\right)+\mu=0
$$

Using the three equalities (8), (9) and (11) we obtain the optimality condition:

$$
\frac{d \mathcal{L}}{d \bar{q}_{1}}=\frac{\partial \mathcal{L}}{\partial \bar{q}_{1}}-\frac{\partial \mathcal{L}}{\partial \bar{q}_{2}}=f_{1}-f_{2}-\left(c_{2}-c_{1}\right)\left[T_{0}+\alpha\left(T_{1}-T_{0}\right)-\alpha \bar{q}_{2} t^{\prime}\left(\bar{q}_{1}\right)\right]=0 .
$$

As a final result, consider the effects of an increase in the MOL of type 2 plants (an increase in $\alpha$ ) on the optimal mix of investment in the two types of plants. Total differentiation of (12) with respect to $\alpha$ leads to:

$$
(1-\alpha) \bar{q}_{2} t^{\prime}\left(\bar{q}_{0}\right)-\bar{q}_{2} t^{\prime}\left(\bar{q}_{1}\right)+\left(T_{1}-T_{0}\right)+\left[(1-\alpha)^{2} t^{\prime}\left(\bar{q}_{0}\right)+2 \alpha t^{\prime}\left(\bar{q}_{1}\right)-\alpha \bar{q}_{2} t^{\prime \prime}\left(\bar{q}_{1}\right)\right] \frac{d \bar{q}_{1}}{d \alpha}=0
$$

Note that unless function $t(q)$ is strongly concave, an increase in $\alpha$, the minimum operating level of type 2 plant, leads to an increase in the optimal investment in type 1 plants. ${ }^{10}$ The intuition is quite simple. An increase in the MOL implies that the system will be operating farther away from the optimal equilibrium without the MOL, and therefore type 2 plants have a higher cost, so that type 1 plants become relatively more attractive.

\footnotetext{
${ }^{9}$ Since $\lambda>0$, equation (6) is also an equality, i.e., it is optimal not to have more capacity than required at peak demand. Equation (7) states that it is optimal not to start operations in plant 2 unless demand exceeds the capacity of plant 1.

${ }^{10}$ Alternatively, the condition can be based on the concavity of $q(t)$.
} 


\subsection{Peak-load pricing}

\subsubsection{A theoretical solution}

Next we extend peak-load pricing to the situation in which MOLs are binding. We begin by setting the price of energy equal to the marginal cost, which is $c_{2}$ in the interval $0-T_{0}$ and $c_{1}$ in the interval $T_{0}-T$. Next we determine the capacity charge. Since the $\lambda$ multiplier represents the cost of a marginal increase in peak demand, from equation (9) it follows that the capacity charge is equal to:

$$
f_{2}^{*}=f_{2}+\left(c_{2}-c_{1}\right) \alpha\left(T_{1}-T_{0}\right)
$$

The MOL, which raises the cost of the system by more than the price of the additional capacity, as additional type 2 capacity is added. The revenues of the two types of plants can be written as:

$$
\begin{aligned}
& R_{1}=c_{2} \int_{0}^{T_{0}} \bar{q}_{1} d t+c_{1} \int_{T_{0}}^{T_{1}}\left\{q(t)-\alpha \bar{q}_{2}\right\} d t+c_{1} \int_{T_{1}}^{T} q(t) d t+f_{2}^{*} \bar{q}_{1} \\
& R_{2}=c_{2} \int_{0}^{T_{0}}\left\{q(t)-\bar{q}_{1}\right\} d t+c_{1} \int_{T_{0}}^{T_{1}} \alpha \bar{q}_{2} d t+f_{2}^{*} \bar{q}_{2}
\end{aligned}
$$

which is sufficient for the type 2 firm to break even and recover the losses it makes by operating between $T_{0}$ and $T_{1}$, and being paid $c_{1}$ per unit of energy delivered. In long run equilibrium, type 1 plants need to break even. Hence

$$
c_{1} \int_{0}^{T_{0}} \bar{q}_{1} d t+f_{1} \bar{q}_{1}=c_{2} \int_{0}^{T_{0}} \bar{q}_{1} d t-f_{2}^{*} \bar{q}_{1}
$$

Rearranging terms, we can rewrite this equality as

$$
f_{1}-f_{2}-\left(c_{2}-c_{1}\right)\left[T_{0}+\alpha\left(T_{1}-T_{0}\right)\right]=0
$$

However this solution is not optimal, as it differs from the optimality condition (13). The reason is quite intuitive. Observe that at any time different from $T_{1}$ and 0 (peak demand), the only effect of a marginal increase in demand is to increase operational costs. At $T_{1}$, however, a marginal increase in demand lengthens the period in which plant 2 operates at its technical minimum by $t^{\prime}\left(\bar{q}_{1}\right)$, and this leads to an increase in on total costs of $-\left(c_{2}-c_{1}\right) \alpha \bar{q}_{2} t^{\prime}\left(\bar{q}_{1}\right)>0$. Formally, since the $\mu$ multiplier represents the cost of a marginal increase in demand at $T_{1}$, from equation (12) it follows it is necessary to impose a capacity charge equal to:

$$
\mu=-\left(c_{2}-c_{1}\right) \alpha \bar{q}_{2} t^{\prime}\left(\bar{q}_{1}\right),
$$

which should be paid only to the type 1 plants as these are the only plants operating at $T_{1}$. As we can see by comparing (12) and (15) this amount is sufficient to provide the right investment signals for type 1 plants. 
Result 2 Peak-load pricing with a MOL requires a capacity charge that is higher than without a MOL and an additional capacity charge paid to low cost firms.

Observe that when there is no indivisibility $(\alpha=0)$, this second capacity charge (16) disappears and the capacity charge (14) becomes the standard capacity charge.

\subsubsection{Implementing peak-load pricing}

In this section we show that peak-load pricing is incompatible with a decentralized system when there are MOLs. To see this point, recall that in the interval $\overline{T_{0} T_{1}}$ plant 2 operates at a loss. In the previous section we showed that it was possible to compensate this plant via a capacity charge that is higher than the marginal cost of capacity. Note however that this requires that plant 2 be compelled to operate, violating the condition that any plant that is dispatched by the system operator obeys willingly, i.e., this is not a decentralized solution. There is an additional problem: at the time of peak demand, users are required to pay the operational cost of the high cost plants, plus the cost of capacity (peak demand $\times$ investment cost of the type 2 plant), plus a surcharge to compensate plant for receiving only $c_{1}$ while operating in $\overline{T_{0} T_{1}}$. Hence there there is an incentive for an entrant to supply all demand at peak load with type two plants, receive the cost of capacity plus the cost of power, plus the additional surcharge (which would give them rents). The problem, of course is that this implies that low cost plants make losses and would exit.

Result 3 Optimal peak-load pricing with a MOL cannot be decentralized.

However, peak-load pricing can be implemented by introducing the rule that capacity will be paid only to plants that generate whenever they are requested to do so. Hence explicit the payment rules would solve the problem, but violate the condition that plants are always willing to generate power when requested. An additional problem lies in the complexity of the solution, which requires the explicit computation of capacity charges by the system operator, negating one of the advantages of the peak load pricing rule in the absence of MOLs.

Up to now we have imposed that the price of energy be set equal to the marginal operational cost. We impose this requirement because if demand were elastic, this would be the requirement of an efficient pricing scheme. In our model however, the load duration curve is independent of price, as is usual in this type of analysis, so the use of marginal cost pricing is not really necessary and is external to the model. Therefore we might as well have incorporated the additional cost caused by the MOL into the price of energy and kept the original capacity charge as the marginal cost of capacity. For instance, we can consider an energy charge equal to $c_{2}$ in period $\overline{T_{0} T_{1}}$. In this case an energy charge above $c_{1}$ is required in $\overline{T_{1} T_{2}}$ in order to achieve the optimal solution. The required energy charge is:

$$
w=c_{1}+\left(c_{2}-c_{1}\right) \frac{\int_{T_{0}}^{T_{1}}[\alpha D-q(t)] d t-\alpha \bar{q}_{2} \bar{q}_{1} t^{\prime}\left(\bar{q}_{1}\right)}{\int_{T_{1}}^{T} q(t) d t} .
$$

However, this solution also faces implementation problems. Note that in the period $\overline{T_{0} T}$ type 1 plants receive more than their marginal cost (since unless $t(q)$ is highly concave, $w>c_{1}$ ). This 
implies that all these plants would like to generate at full capacity, and hence supply would exceed demand. Hence, the effective demand faced by these plants must be assigned proportionally to the capacity of each plant.

\section{Conclusions}

This paper has shown that when plants have minimum operating levels, the standard peak-load pricing system must be modified in order to achieve the (long run) optimal investment mix between different plants. It has also shown that the solution cannot be implemented via a decentralized mechanism. In the special case where demand is inelastic, the optimal solution can also be implemented by raising the energy price.

MOLs are important in deregulated marginal cost dispatch systems, where the use of the standard peak-load pricing formulas can lead to inefficiencies. The fact that the legislation in the countries that use peak load pricing does not cover these and other imperfections means that firms must use informal methods of settling these problems. However, this also implies that a new entrant faces unwritten rules, which might be one of the reasons for the lack of entry into the electric markets in these countries.

There are other problems that involve similar issues and which could be analyzed by analogous methods: for example, the long ramp-up times of some plants mean that high cost plants are sometimes required to operate as base-load plants, creating inversions in the merit order. We have made a strong simplification in our analysis, as we have assumed that demand is constant and does not respond to the existence of an additional power charge. Removing this restriction is another topic for future research. ${ }^{11}$

\footnotetext{
${ }^{11}$ As in Oren et al. (1985).
} 


\section{References}

Boiteux, M. (1960). Peak load pricing. The Journal of Business, 33, 157-179.

Chao, H.-P. (1983). Peak load pricing and capacity planning with demand and supply uncertainty. The Bell Journal of Economics, 14(1), 179-190.

Joskow, P. L. (1976). Contributions to the theory of marginal cost pricing. The Bell Journal of Economics, 7(1), 197-206.

Oren, S., Smith, S., and Wilson, R. (1985). Capacity pricing. Econometrica, 53(3), 545-566.

Turvey, R. (1968). Optimal Pricing and Investment in Electricity Supply. MIT Press, Cambridge, MA.

Williamson, O. E. (1966). Peak-load pricing and optimal capacity constraints under indivisibility constraints. The American Economic Review, 56(4), 810-827. 